\title{
Heightened stress responsivity and emotional reactivity during pubertal maturation: Implications for psychopathology
}

\author{
LINDA PATIA SPEAR \\ Binghamton University
}

\begin{abstract}
This commentary reviews and reflects on the studies of this special section: studies that collectively provide compelling evidence for meaningful changes in stress- and emotionally reactive psychophysiological systems with the transition from middle childhood into adolescence. The observed changes were complex and often overlaid upon ontogenetic differences in basal levels of activation of these systems. Maturational increases in responsiveness to stressors were stressor dependent and differentially expressed across autonomic and hormonal measures. Pubertal status increased the impact of some affective valence manipulations, although not significantly influencing others, including negative affect-related potentiation of startle/reflexes. Such ontogenetic increases in stressor and affect sensitivity may have implications for developmental psychopathology. Developmental increases in stressor reactivity may normally aid youth in responding adaptively to the challenges of adolescence, but may result in stress dysregulation among at-risk adolescents, increasing further their vulnerability for psychopathology. Pubertalrelated increases in sensitivity to emotionally laden stimuli may exacerbate individual predispositions for exaggerated affective processing, perhaps contributing to the emergence of psychological disorders in these youth. Together, these studies, with their innovative use of autonomic, reflexive, and hormonal measures to index age- and pubertal-related changes in reactivity to stressors and affective stimuli, provide promising directions for future research. Some of these, along with a few cautionary notes, are outlined.
\end{abstract}

I am delighted to have been invited to provide a commentary on this special section of Development and Psychopathology that focuses on changes in stress reactivity and sensitivity to emotional stimuli during the transition from middle childhood to adolescence. I was particularly pleased to have been asked to comment on the papers in this special section because in previous reviews (e.g., Spear, 2000, 2003) I had proposed that normative developmental alterations in the neurobiology of stress and emotional reactivity might increase stressor reactivity during the pubertal/adolescent transition, precipitating the emergence of psychopathology among vulnerable individuals and thereby contributing to increases in affective and other psychological disorders seen between childhood and adolescence. Similar suggestions have been made by others (e.g., Dahl, 2004; Walker, Sabuwalla, \& Huot, 2004). Few studies have provided data relevant to the evaluation of these hypotheses. Of particularly importance would be studies of normally developing children to determine whether the childhood to adolescent transition is indeed characterized by developmental changes in the physiology of stress and emotional reactivity. This special section provides four such studies. Collectively, these studies provide clear, convincing, and compelling evidence for such changes in typically developing children, changes that are

Copyright () 2009 Cambridge University Press

Address correspondence and reprint requests to: Linda Patia Spear, Department of Psychology and Center for Development and Behavioral Neuroscience, Binghamton University, Binghamton, NY 13902-6000; 1spear@binghamton.edu.. 
often influenced by pubertal status and differentially expressed across various autonomic and reflex measures. This commentary summarizes and discusses various reoccurring themes and issues in these studies, raising a few cautionary notes and proposing several promising future research directions leading from these seminal experiments.

In the study by Gunnar, Wewerka, Frenn, Long, and Griggs in this issue (2009), baseline and stress-related indices of cardiac and hypothalamo-pituitary-adrenal (HPA) axis function were examined in 9- to 15-year-old males and females tested using a child-adapted version of the Trier Social Stress Test (TSST). Developmental increases in both basal cortisol and stress-related elevations in cortisol were seen, with the latter emerging earlier in females than males. Intriguingly, analysis of the cardiac measures revealed not only typical developmental decreases in basal heart rate, but also a developmental decline in stressorrelated sympathetic activation, with no age-related changes evident in indices reflecting parasympathetic tone.

Developmental increases in stressor responsiveness during adolescence were also observed by Stroud et al. (this issue, 2009). In that study, a number of autonomic measures reflecting cardiac and HPA axis function were assessed in 7- to 12-year-old and 13- to 17-year-old individuals prior to, during, and following either a social (peer rejection) stressor or performance-related stressors that included public speaking, mental arithmetic, and mirror tracing. Greater responsiveness to both stressors was seen in the older group, although the specific physiological measures where age differences emerged varied with stressor type.

Silk and colleagues (this issue, 2009) studied 8- to 18-year-olds and compared pre-/early pubescent and mid-/late pubescent males and females in terms of their pupillary reactivity to words with positive, negative, and neutral valences during a task where participants were asked to attribute the appropriate valence to each of the words, and later were given an unexpected recall test for the words. Individuals in the mid/late pubertal group remembered more emotional words (especially those with negative valence) than the pre-/early puberty group, and also rated themselves as more emotional (especially in terms of negative affect) during the laboratory visit. The mid-/late pubertal group also showed greater peak pupillary reactivity to the words and more rapid postword decline of the pupillary dilation response, effects evident regardless of word valence, and hence, potentially reflecting general increases in processes involving pupillary control.

Pubertal influences on sensitivity to stimuli of different affective valence were also examined by Quevedo, Benning, Gunnar, and Dahl (this issue, 2009) via assessing the efficacy of pleasant, neutral, and aversive pictures for modulating eye-blink startle and an auditory-associated motor reflex. Along with a developmental increase in startle amplitude, with greater startle responses regardless of picture valence in the postpubertal than prepubertal group, only post-pubertal individuals showed appetitive potentiation of the auditory motor reflex.

In addition to providing converging evidence for alterations in stress- and affect-responsivity during adolescence and the pubertal period, these studies also raise a number of reoccurring themes and issues, several of which are detailed below.

\section{Affect-Modulated Psychophysiological Responses and Puberty}

The Silk et al. (2009) and Quevedo et al. (2009) studies in this issue combine assessment of affect-related modulation of motor reflexes (pupillary reactivity, eye-blink startle, an auditory motor reflex) with self-report, questionnaire, or task performance data to index effects of pubertal status on hedonic responsiveness and emotional reactivity to stimuli of different valences. These studies largely forge new ground: the strategy of using affect 
modulation of startle and reflexes to probe emotional reactivity has previously received scant attention in developmental studies, despite the profitable use of such approaches in adulthood (e.g., Lang, Bradley, \& Cuthbert, 1990).

Pubertal effects emerged in both the Silk et al. (this issue, 2009) and Quevedo et al. (this issue, 2009) studies, albeit not always in terms of alterations in affect-modulated startle/ reflexes per se. Silk et al. found pubertal status to interact with word valence in the recall data, with mid-/late pubertal individuals (but not those who were less pubertally mature) remembering more emotional than nonemotional words. In the Quevedo et al. study, evidence for pubertally related affect modulation was seen in the auditory motor reflex, with appetitive potentiation of this reflex seen in postpubertal individuals, but not among prepubertal participants. Quevedo and colleagues also found pubertal status to influence expression of individual differences in the processing of emotional stimuli, with behavioral trait characteristics correlated with greater affect modulation of startle and the auditory motor reflex only among post-pubertal and not prepubertal individuals.

Perhaps as interesting, and ultimately as informative, as the puberty-related changes in affect measures seen in these studies were those indices of affect reactivity that proved resistance to pubertal change. Increases in startle/reflex magnitude with negative valence stimuli were not related to pubertal status in either study. In the Silk et al. (this issue, 2009) study, puberty-related changes in pupillary reactivity were not influenced by valence of the test stimuli, with mid-/late pubertal individuals showing greater overall peak pupillary reactivity and a more rapid postword recovery than the pre-/early pubertal group regardless of word valence. Similarly, in the Quevedo et al. (this issue, 2009) study, although negative affect was found to potentiate the eye-blink startle response, this affect-modulated effect was unrelated to pubertal status, with general puberty-related increases in overall startle amplitude regardless of stimulus valence. Thus, although pubertal-related changes in overall startle/reflex parameters often emerged in these studies, along with some evidence for affect modulation of these reflexes, pubertal status often did not interact with the affect valence manipulations. Together, these studies provide evidence for pubertal-related alterations in basic startle/reflex circuitry without notable changes in negative affect-induced potentiation of these startle/reflex measures, although pubertal status by valence interactions did emerge with other outcome measures. Given evidence that neuro-circuitry underlying basic startle/ reflexes varies from that contributing to negative affect-related startle potentiation (e.g., fear-potentiated startle; see Bradley, Lang, \& Cuthbert, 1993; Rosen \& Schulkin, 1998), these findings may provide intriguing clues as to pubertal-sensitive and pubertal-insensitive neural systems engaged in and influenced by the processing of emotional stimuli during the transitions of adolescence.

\section{Adolescent-Associated Increases in Stressor Sensitivity}

Both the Gunnar et al. (this issue, 2009) and Stroud et al. (this issue, 2009) articles used multiple psychophysiological measures as probes to index stressor reactivity during adolescence. In both studies some remarkably similar developmental increases in responsiveness to stressors were seen. For instance, both found ageand puberty-related increases in stress reactivity to a performance stressor task (TSST-C or modified TSST-C) when cortisol was used to index stressor-induced activation of the HPA. Additional acrossstudy similarities were seen in terms of age-related increases in regulation of sympathetic reactivity, with evidence of greater sympathetic regulation among older (pubertal) individuals seen in the Gunnar et al. study and evidence for an age-related decline in heart rate accompanied by increased cardiac reactivity to stressors in the work of Stroud et al. 
These studies also found that age- and puberty-dependent increases in stressor reactivity were sometimes measure and stressor specific. For instance, the age-associated increase in stressor-related HPA activation found by Stroud and colleagues (this issue, 2009) on the performance stressor task was not evident with the peer rejection stressor. In terms of measure dependency, whereas Stroud et al. found developmental increases in autonomic activation in response to both the performance and peer-rejection stressors, the specific autonomic measures showing ontogenetic increases were stressor specific (i.e., evident with diastolic blood pressure [BP] in the performance stress situation, but with systolic BP and saliva $\alpha$-amylase under circumstances of peer rejection stress). These results speak to the importance of assessing multiple psychophysiological measures in ontogenetic studies of stressor sensitivity, as well as determining possible stressor-specific effects.

The sometimes stressor-specific nature of the age- or puberty-related endocrine and autonomic findings obtained in the Gunnar et al. (this issue, 2009) and Stroud et al. (this issue, 2009) articles also point to possible intriguing differences in the psychophysiological systems activated by stressors during ontogeny. Stressors and other affective challenges activate a number of centrally modulated psychophysiological systems that, through coordinated and sometimes complementary effects (e.g., see Bauer, Quas, \& Boyce, 2002), normally serve to mobilize and prepare the body to meet the challenges presented. Activation of stress-responsive systems, such as the sympathetic and parasympathetic (vagal) arms of the autonomic nervous system (ANS) and the HPA axis, often produce similar net effects, but accomplish these effects differently, with separable costs/benefits. For example, stress-induced increases in heart rate can be induced by the ANS either via sympathetic activation or parasympathetic withdrawal, although heart rate increases stimulated via downregulation of parasympathetic tone (vagal breaking) have been suggested to be more rapidly reversible and less metabolically costly than heart rate increases induced via sympathetic activation (see Porges, 2001). Stress-induced activation of the HPA axis has a slower onset and recovery than activation of the ANS, and may show more adaptation with repeated stressors than the ANS response (e.g., Gordis, Granger, Susman, \& Trickett, 2008). These different stress-responsive systems also differ in their phylogeny, their interaction with the immune and other physiological systems (Porges, 2001), and their central regulation (e.g., Radley, Arias, \& Sawchenko, 2006).

Asymmetries between ANS and HPA activation in response to stressors have been reported among youth with behavior problems (Bauer et al., 2002; Gordis, Granger, \& Susman, 2006) as well as among maltreated youth (Gordis et al., 2008), and have been suggested to be partially protective against the adverse effects of chronic stress during ontogeny. The results of Gunnar et al. (this issue, 2009) and Stroud et al. (this issue, 2009) suggest that these stress-sensitive autonomic and neuroendocrine systems and the relative balance among them also differ in their ontogeny. Similar examples can be seen in the animal literature (e.g., Kurtz \& Campbell, 1994; Lyss, Andersen, LeBlanc, \& Teicher, 1999). Detailing the dynamic interplay between normative developmental patterns of stress-induced ANS/HPA activation and ANS/HPA asymmetries already present in certain at-risk youth as they enter the transitions of adolescence and puberty may provide important clues regarding their vulnerability for the development of psychopathology during these transitions.

Findings of developmental differences in ANS and HPA reactivity to stressors and other emotional challenges during pubertal/adolescent periods perhaps should not be surprising given that limbic and prefrontal areas known to be critical for regulating and sensing these physiological systems (regions such as the amygdala, ventral striatum, insula, prefrontal and anterior cingulate cortices; e.g., Craig, 2002; Dalgleish, 2004) undergo considerable developmental change during adolescence (see Nelson, Leibenluft, McClure, \& Pine, 2005). For an example, using a simple animal model of adolescence in the rat, Lyss and colleagues 
(1999) found that stress induced by an anxiogenic, beta-carboline drug predominantly resulted in prefrontal activation in postpubertal rats, but primarily induced activation in ventral striatum (nucleus accumbens) among prepubertal animals. Developmental differences in the propensity to recruit these neural control systems in stressor-specific ways to influence their somatic expression could ultimately have critical implications for understanding how adolescents respond to emotional and stressful situations. Ontogenetic differences in environmentally reactive psychophysiological systems may be particularly relevant for understanding adolescent emotional responses given evidence, albeit mixed, for emotion-specific patterns of somatic activation (Collet, VernetMaury, Delhomme, \& Dittmar, 1997) that serve as "emotion-based biasing signals" that can be used as feedback cues for self-attribution of affect (see Dunn, Dalgleish, \& Lawrence, 2006, for critique and discussion). Results presented in this special section hint to the provocative possibility that this peripheral contribution to affect attribution could perhaps be more subtle among adolescents than previously reported in adults. That is, the developmental changes Stroud et al. (this issue, 2009) observed in physiological responses to the stressors "were not mirrored by differences in affective responses to the stressors," despite a nonsignificant tendency for adolescents to show more negative affect and less positive affect than younger individuals in both stressor situations. A similar dissociation was also noted by Gunnar and colleagues (this issue, 2009). Whether adolescents not only show different patterns of somatic responding to stressors and other challenges, but also differ from more mature individuals in their likelihood to use that information for emotional attribution provide intriguing questions for future study.

Taken together, the results of Gunnar et al. (this issue, 2009) and Stroud et al. (this issue, 2009) provide convincing evidence for increases in stressor reactivity during the transition from middle childhood to adolescence, along with data demonstrating that the ontogeny of stress-induced psychophysiological responses likely reflects complex interactions between the nature of the stressor, the autonomic/endocrine system under investigation and its neural regulators and sensors, as well as the developmental and pubertal state of the individual. Further assessment of patterns of somatic activation in response to stressors and other challenges during puberty and the broader adolescent period is clearly an important area for further study, especially given the potential involvement of these peripherally generated signals in influencing affect attribution (e.g., Craig, 2002), as well as in contributing to the vulnerability to behavior disorders such as drug abuse (e.g., Piazza \& LeMoal, 1996).

\section{Maturation-Related Differences in Basal Psychophysiological Activity}

Maturational changes in basal measures of psychophysiological functioning are often seen among children and adolescents. Indeed, in the Gunnar et al. (this issue, 2009) and Stroud et al. (this issue, 2009) studies, developmental increases were seen in basal cortisol levels with age and pubertal status, along with characteristic ontogenetic declines in heart rate. Maturational differences in "resting" levels of these stress-sensitive measures highlight the importance of determining baseline, nonstressed levels of each measure prior to stressor challenge. That is, if prechallenge "basal" levels taken in the test context actually reflect not only true basal differences in these measures but also some degree of stress-induced elevation that varies with age/pubertal status, such elevated baselines could obscure detection of age- or pubertal-related differences in responsivity to the target stressors. The Gunnar et al. and Stroud et al. studies presented in this special section provide two different strategies for addressing potential baseline issues. Gunnar et al. obtained data on basal HPA levels at home as well as in the laboratory, convincingly demonstrating that cortisol obtained under resting conditions after arrival in the laboratory may be elevated from that seen with samples collected at home. Stroud et al. brought participants into the laboratory twice, using the first visit to acclimatize them to the laboratory setting prior to collecting data following 
the target experimental stressors on the second visit. Both approaches are valuable strategies for addressing the potential intrusion of the stressfulness of the basic laboratory experience into baseline assessments of stressor-sensitive dependent measures.

A somewhat analogous situation could potentially emerge when indexing age- or pubertalrelated differences in affect-modulation of reflex and startle reactivity. For instance, to the extent that baseline affect is influenced by pubertal status or age (e.g., see Larson \& Lampman-Petraitis, 1989), this underlying affect bias could influence overall startle reflex reactivity during the test, with these alterations perhaps obscuring detection of affectspecific alterations across test stimuli with different valences. For example, individuals in the mid-/late puberty group in the Silk et al. (this issue, 2009) study rated themselves higher in current negative affect (as indexed via the Positive and Negative Affect Schedule for Children) and reported themselves as more emotional during the test than the pre-/early puberty group; this mid-/late pubertal group also showed greater peak pupillary dilation during testing than the pre-/early pubertal group, regardless of word valence. It is also interesting that the only reflex startle measure that showed reliable puberty-dependent affect modulation in the studies of this special section (i.e., the auditory motor reflex in the study by Quevedo et al., this issue, 2009) was also the only measure devoid of pubertal or agerelated differences in overall reflex magnitude. Of course, maturation-related changes in baseline startle reflex responsiveness may be influenced not only by differences in basal affect levels within the test situation, but also by developmental differences in motor functions and their neural control. Although baseline differences across age or pubertal status can be controlled statistically to some extent (see, e.g., Silk et al.), careful consideration of potential baseline differences is likely to continue to be critical when using hormonal, autonomic, and startle/reflex measures to assess age differences in reactivity to stressful and emotional stimuli.

\section{Implications for Studies of Developmental Psychopathology}

Findings from the studies of this special section showing adolescent- and/or pubertalassociated increases in sensitivity to stressors and affect-laden stimuli may have implications for developmental psychopathology. The adolescent period is associated with notable increases in the incidence of a variety of psychological disorders, including depression, social anxiety, eating disorders, substance abuse/dependence, and schizophrenia (Kessler et al., 2005). Among the factors postulated to contribute to this rise in psychopathology during adolescence are maturationally related increases in the sensitivity of adolescents to stressors and socioemotional stimuli (e.g., Steinberg et al., 2006; Walker et al., 2004). For most adolescents, increases in stress and affect responsivity have been suggested to help the adolescent attend and respond appropriately to increasingly diverse environmental circumstances. Yet, among at-risk youth, these developmental amplifications in reactivity may increase their vulnerability to psychopathology. For instance, as proposed earlier (Spear, 2000), normative increases in HPA and autonomic responses to stress during adolescence may normally aid adolescents in responding adaptively to the challenges of adolescence (see also Gunnar et al., this issue, 2009; Stroud et al., this issue, 2009), although among "high-risk adolescents, this normative shift may tip the balance toward stress response dysregulation associated with depression and other psychopathology" (Stroud et al.). How this shift toward dysregulation of the stress response might be expressed in at-risk individuals remains to be determined; as discussed by Stroud et al., possibilities include atypical reactions to particular stressors, exaggerated reactivity across multiple stressors, and an altered balance of stress-induced autonomic/HPA reactions. In terms of the processing of affective stimuli, pubertal-related increases in emotional sensitivity has been suggested to enhance individual predispositions in affective processing, with Quevedo et al. (this issue, 2009) showing that anxious individuals become even more sensitive to anxiety-provoking 
stimuli following puberty. Likewise, when probing stress reactivity as well, individuals high on temperament ratings of anxiety showed greater stress-induced increases in cortisol among girls (Gunnar et al.).

The role of individual differences in influencing maturational increases in stress and affect responsivity, such as those seen in the studies of Gunnar et al. (this issue, 2009) and Quevedo et al. (this issue, 2009), provide support for exploring the interplay between temperament or trait variables, maturational increases in stress and affect responsivity, and the emergence of psychological disorders in at-risk individuals. Are vulnerable youth prone to exhibit different ontogenetic patterns of physiological and affective reactions to challenges? Or do they display normative changes, but either respond atypically to them or are bombarded by more (perhaps self-exacerbated) life challenges? Do certain developmental patterns of autonomic/HPA responses to stressors and emotional stimuli help confer resistance to resilient youth? As work exemplified by this special section detail normal developmental patterns of responsiveness to stressors and affective stimuli, such research can be extended to examine these dynamics in children at risk for psychopathology.

\section{Future Directions}

There are many promising directions leading from the work presented in this special section, some of which were discussed earlier. Others, along with a few cautionary notes, are briefly detailed here.

\section{Further studies of puberty}

Instances of pubertal influences on stress reactivity and responsiveness to emotionally relevant stimuli are scattered throughout the special section, although as discussed above, some measures of affect reactivity seemed relatively resistant to pubertal change. Clearly, much remains to be determined about the role of puberty in adolescent-related neurobiological changes in stressor sensitivity and emotional reactivity. Psychophysiological assessments such as those used in these studies provide an exciting, informative, and previously underutilized developmental strategy to probe reactivity to stressors and affective stimuli during puberty, and offer a critical complement to neurophysiological and imaging studies examining emotion regulation and recognition during development (e.g., Lewis, Lamm, Segalowitz, Stieben, \& Zelazo, 2006; Monk et al., 2003). As work in this area continues to detail processes that are associated with pubertal progress and those that are not, focus can then be directed toward determining what specific components of puberty are critical for these associations. Future work in this area would be facilitated by longitudinal studies, as well as by more detailed measures of pubertal progress, including multihormonal assessments and other physiological indices; see Angold, 2003).

A longitudinal approach that begins prior to pubertal onset may prove important to distinguish effects associated with state of pubertal maturation per se from those related to pubertal timing. This is important in that entering puberty early or late relative to one's peers is a known risk factor for disrupted social and psychological functioning and the emergency of psychopathology (see Waylen \& Wolke, 2004; Zehr, Culbert, Sisk, \& Klump, 2007). For instance, early puberty in girls is associated with an increase in a variety of adverse outcomes, including elevated expression of symptoms of depression (Ge, Conger, \& Elder, 2001; Ge et al., 2003), anxiety, and eating disorders (Zehr et al., 2007). The timing of pubertal onset is only poorly correlated, however, with the tempo (rate of progression) of puberty (Biro, Abrahams, Post, \& Parkinson, 2006). Thus, cross-sectional studies that rely on estimates of current pubertal status among young adolescents seemingly would include within their pubertal groups both individuals with early pubertal onset as well as those with normal timed puberties, potentially confounding interpretation of data related to pubertal 
status per se. Although longitudinal studies are best able to distinguish pubertal timing from current pubertal status, it seemingly would be feasible even in cross-sectional studies to obtain rough estimates of the timing of pubertal onset to help distinguish the contribution of this factor from pubertal status per se.

With the current focus on parsing which adolescent-typical neurobiological and emotional/ behavioral changes are and are not pubertally linked (e.g., see Nelson et al., 2005), it may prove useful in cross-sectional work to examine not only individuals of similar chronological age that vary considerably in pubertal status (see the study by Quevedo et al. for an excellent use of this strategy, this issue, 2009), but also to examine individuals of similar pubertal status that vary notably in chronological age. From a statistical perspective, associations between a particular variable and outcome measures are more likely to emerge when the variable is allowed to vary widely than when the range of variable expression is limited (e.g., see Martin, Ballard, \& Higgins, 2002). Indeed, in studies involving samples where both pubertal status and chronological age vary notably, pubertal status is typically correlated highly with chronological age (e.g., Booth, Johnson, Granger, Crouter, \& McHale, 2003), with both measures perhaps serving in part as general proxies for overall developmental pace. Body weight per se is strongly linked to pubertal timing, with heavier individuals entering puberty earlier than those of lighter weight (Karlberg, 2002; Roemmich, Richmond, \& Regol, 2001), an effect particularly pronounced in females where body size/ composition may convey evidence regarding the sufficiency of energy stores to support a pregnancy and lactation (Frisch, 1991). As such, it is possible that puberty might even serve as a more reliable index of overall maturational rate than chronological age per se.

In addition to potentially serving as a proxy for general developmental pace, puberty also may be directly associated with specific neural alterations. Certain brain transformations, particularly in the hypothalamus and related regions modulating gonadotropin release, are critical for precipitating the pubertal process. As levels of gonadal hormones and other pubertally sensitive hormones rise during puberty, they may selectively bind to hormone receptors in hormone-receptive brain regions (for a review, see Nelson et al., 2005), thereby exerting "activational" effects that require the ongoing presence of gonadal hormones for their expression (e.g., see Sisk \& Zehr, 2005). There is also compelling evidence from studies with laboratory animals that, in addition to the well-known, gonadal hormonedirected sexual differentiation of the brain during the embryonic and early postnatal period, rising titers of hormones at puberty may induce a second "organizational" period, serving to guide the remodeling of the adolescent brain in sex-appropriate ways (for a review, see Sisk $\&$ Zehr, 2005). Little is yet known of how pubertal processes, including organizational and activational effects of pubertal hormones, contribute to neural, emotional, and cognitive changes during adolescence. Progress in this area may be aided by basic research to assess consequences of manipulating hormone levels in laboratory animals at specific points during juvenile/adolescent/ adult transitions.

\section{Focus on sex differences}

Basic research using laboratory animals has revealed that pubertally related activational and organizational influences of gonadal hormones are prototypically sexually dimorphic (see Sisk, Schulz, \& Zehr, 2003). Hence, to the extent that pubertal-emergent neurobehavioral changes seen in human adolescents reflect similar influences, emerging sex differences would also seem likely. Transient sex differences might also emerge at certain points in adolescence, given relative differences between the sexes in developmental timing, with females typically undergoing pubertal changes earlier than males (e.g., Karlberg, 2002). Data from the Gunnar et al. (this issue, 2009) study in this special section provides an excellent example of a transiently appearing sex difference that could potentially reflect such a timing effect, with developmental increases in basal cortisol levels and stress-related 
increases in cortisol emerging earlier in females than in males. In contrast, in the Quevedo et al. (this issue, 2009) study, where sex was included as grouping factor, no significant main effects or interactions involving sex were seen.

Despite a rising number of reports from imaging studies of emergent sex differences in adolescent brain (e.g., Canli, Desmond, Zhao, \& Gabrieli, 2002; Cosgrove, Mazure, \& Staley, 2007; Lenroot et al., 2007), there has been little emphasis on detailing sex-related changes in psychophysiological responses to stressors and other emotional challenges during puberty (e.g., see studies in this section by Silk et al., 2009; Stroud et al., 2009). This may be in part because in these initial investigations of stress/affect/puberty interrelationships, the focus has largely been on other critical modulating variables (e.g., personality/behavioral traits), with the consequence that experiments may not have been sufficiently powered to reveal potential sex differences. Careful assessment of sexually dimorphic pubertal effects remains an important area for future focus, especially given the well-characterized emergence of sex differences in psychological disorders such as depression during adolescence (e.g., Nolen-Hoeksema \& Girgus, 1994).

\section{Other potentially important moderating factors: Ethnicity and economic circumstances}

At this early stage in the study of stress- and affect-sensitive neurobiological changes during adolescence, the studies in this special section have focused on normative, low-risk populations of youth. Indeed, the children in these studies were principally Caucasian, drawn largely from relatively privileged, middle-income or greater family backgrounds, and generally were screened to remove those with medical or psychological disorders. It is critical to determine the generality of these studies by extending such research to youth from other ethnicities and life settings (especially contexts involving poverty and other riskconferring circumstances), and to youth with known risk factors such as histories of prior stress or abuse. Such studies may provide intriguing complexities. Early pubertal timing is characteristic of some ethnicities (such as African Americans; e.g., Kaplowitz, 2006) and certain stressful life circumstances (e.g., Ellis \& Garber, 2000; for a review, see Ellis, 2004). Moreover, there are reports of age-related differences in the impact of socioeconomic status (SES) on physiology and health with, for instance, low SES associated with increased basal cortisol levels in children but not in adolescents (Lupien, King, Meaney, \& McEwen, 2001). Indeed, there are long-standing data documenting SES-related health inequities during childhood that shift to relative pariety during adolescence before SES inequities reemerge in adulthood (West, 1997). Discerning how normal developmental and pubertal changes in physiological reactivity to stressors and other affective stimuli interact with early experience-, ethnic-, context-, and temperament-associated alterations in these same measures to affect the probability of adolescent-emergent psychopathology is challenging, but arguably essential to determine why "mental disorders . . . have their strongest foothold in youth" (Kessler et al., 2005, p. 601).

\section{Summary and Conclusions}

Affective reactions color our perceptions and influence the way we respond and adapt to daily life events. The work described in this special section provides convincing evidence for normative pubertal and maturational increases in reactivity to stressors and emotionally valenced stimuli, along with hints as to intriguing complexities regarding how and under what circumstances these ontogenetic changes are expressed. Through innovative use of autonomic, reflexive, and hormonal measures to index pubertal- and maturational-related changes in reactivity to stressors and affective stimuli, the studies of this special section are at the forefront of research exploring how adolescents react to meet the challenges associated with their increasingly complex life experiences. These psychophysiological approaches provide a fertile approach for future research exploring circumstances under 
which dynamic developmental and pubertal changes in stressor and affect reactivity during adolescence promote or disrupt adaptations among resilient and at-risk youth living in "scaffolded" versus unconstructive environments, perhaps contributing to the emergence of psychopathology among the most vulnerable.

\section{Acknowledgments}

Preparation of this MS was supported by NIDA Grant DA019071 and NIAAA Grants R01 AA-16887, R01 AA017355, and R37 AA 12525.

\section{References}

Angold A. Adolescent depression, cortisol and DHEA [Editorial]. Psychological Medicine 2003;33:573-581. [PubMed: 12785459]

Bauer AM, Quas JA, Boyce WT. Associations between physiological reactivity and children's behavior: Advantages of a multisystem approach. Journal of Developmental and Behavioral Pediatrics 2002;23:102-113. [PubMed: 11943973]

Biro PA, Abrahams MV, Post JR, Parkinson EA. Predators select against high growth rates and risktaking behaviour in domestic trout populations. Proceedings of the Royal Society of London Series B 2004;271:2233-2237. [PubMed: 15539348]

Booth A, Johnson DR, Granger DA, Crouter AC, McHale S. Testosterone and child and adolescent adjustment: The moderating role of parent-child relationships. Developmental Psychology 2003;39:85-98. [PubMed: 12518811]

Bradley MM, Lang PJ, Cuthbert BN. Emotion, novelty, and the startle reflex: Habituation in humans. Behavioral Neuroscience 1993;107:970-980. [PubMed: 8136072]

Canli T, Desmond J, Zhao Z, Gabrieli JD. Sex differences in the neural basis of emotional memories. Proceedings of the National Academy of Sciences of the United States of America 2002;99:1078910794. [PubMed: 12145327]

Collet C, Vernet-Maury E, Delhomme G, Dittmar A. Autonomic nervous system response patterns specificity to basic emotions. Journal of the Autonomic Nervous System 1997;62:45-57. [PubMed: 9021649]

Cosgrove KP, Mazure CM, Staley JK. Evolving knowledge of sex differences in brain structure, function, and chemistry. Biological Psychiatry 2007;62:847-855. [PubMed: 17544382]

Craig AD. How do you feel? Interoception: The sense of the physiological condition of the body. Nature Reviews Neuroscience 2002;3:655-666.

Dahl RE. Adolescent brain development: A period of vulnerabilities and opportunities. Annals of the New York Academy of Sciences 2004;1021:1-23. [PubMed: 15251869]

Dalgleish T. The emotional brain. Nature Reviews Neuroscience 2004;5:582-589.

Dunn BD, Dalgleish T, Lawrence AD. The somatic marker hypothesis: A critical evaluation. Neuroscience and Biobehavioral Reviews 2006;30:239-271. [PubMed: 16197997]

Ellis BJ. Timing of pubertal maturation in girls: An integrated life history approach. Psychological Bulletin 2004;130:920-958. [PubMed: 15535743]

Ellis BJ, Garber J. Psychosocial antecedents of variation in girls' pubertal timing: Maternal depression, stepfather presence, and marital and family stress. Child Development 2000;71:485-501. [PubMed: 10834479]

Frisch, RE. Puberty and body fat. In: Lerner, RM.; Petersen, AC.; Brooks-Gunn, J., editors. Encyclopedia of adolescence. Garland; New York: 1991. p. 884-892.

Ge X, Conger RD, Elder GH Jr. Pubertal transition, stressful life events, and the emergence of gender differences in adolescent depressive symptons. Developmental Psychology 2001;37:404-417. [PubMed: 11370915]

Ge X, Kim I, Brody G, Conger RD, Simons RL, Gibbons F, et al. It's about timing and change: Pubertal transition effects on symptoms of major depression among African American youths. Developmental Psychology 2003;39:430-439. [PubMed: 12760513] 
Gordis EB, Granger DA, Susman EJ, Trickett PK. Asymmetry between salivary cortisol and $\alpha$ amylase reactivity to stress: Relation to aggressive behavior in adolescents. Psychoneuroendocrinology 2006;31:976-987. [PubMed: 16879926]

Gordis EB, Granger DA, Susman EJ, Trickett PK. Salivary alpha amylase-cortisol asymmetry in maltreated youth. Hormones and Behavior 2008;53:96-103. [PubMed: 17945232]

Gunnar MR, Wewerka S, Frenn K, Long JD, Griggs C. Developmental changes in HPA activity over the transition to adolescence: Normative changes and associations with puberty. Development and Psychopathology 2009;21:69-85. [PubMed: 19144223]

Kaplowitz P. Pubertal development in girls: Secular trends. Current Opinion in Obstetrics and Gynecology 2006;18:487-491. [PubMed: 16932041]

Karlberg J. Secular trends in pubertal development. Hormone Research 2002;57(Suppl. 2):19-30. [PubMed: 12065922]

Kessler RC, Berglund P, Demler O, Jin R, Merikangas KR, Walters EE. Lifetime prevalence and ageof-onset distributions of dsm-iv disorders in the national comorbidity survey replication. Archives of General Psychiatry 2005;62:593-602. [PubMed: 15939837]

Kurtz MM, Campbell BA. Paradoxical autonomic responses to aversive stimuli in the developing rat. Behavioral Neuroscience 1994;108:962-971. [PubMed: 7826518]

Lang PJ, Bradley MM, Cuthbert BN. Emotion, attention, and the startle reflex. Psychological Review 1990;97:377-395. [PubMed: 2200076]

Larson R, Lampman-Petraitis C. Daily emotional states as reported by children and adolescents. Child Development 1989;60:1250-1260. [PubMed: 2805902]

Lenroot RK, Gogtay N, Greenstein DK, Wells GL, Clasen LS, Blumenthal JD, et al. Sexual dimorphism of brain developmental trajectories during childhood and adolescence. NeuroImage 2007;36:1065-1073. [PubMed: 17513132]

Lewis M, Lamm C, Segalowitz SJ, Stieben J, Zelazo PD. Neurophysiological correlates of emotion regulation in children and adolescents. Journal of Cognitive Neuroscience 2006;18:430-443. [PubMed: 16513007]

Lupien SJ, King S, Meaney MJ, McEwen BS. Can poverty get under your skin? Basal cortisol levels and cognitive function in children from low and high socioeconomic status. Development and Psychopathology 2001;13:653-676. [PubMed: 11523853]

Lyss PJ, Andersen SL, LeBlanc CJ, Teicher MH. Degree of neuronal activation following fg-7142 changes across regions during development. Brain Research. Developmental Brain Research 1999;116:201-203. [PubMed: 10521564]

Martin JR, Ballard TM, Higgins GA. Influence of the 5-ht2c receptor antagonist, sb-242084, in tests of anxiety. Pharmacology, Biochemistry, and Behavior 2002;71:615-625.

Monk CS, McClure EB, Nelson EE, Zarahn E, Bilder RM, Leibenluft E, et al. Adolescent immaturity in attention-related brain engagement to emotional facial expressions. NeuroImage 2003;20:420428. [PubMed: 14527602]

Nelson EE, Leibenluft E, McClure E, Pine DS. The social re-orientation of adolescence: A neuroscience perspective on the process and its relation to psychopathology. Psychological Mediciine 2005;35:163-174.

Nolen-Hoeksema S, Girgus JS. The emergence of gender differences in depression during adolescence. Psychological Bulletin 1994;115:424-443. [PubMed: 8016286]

Piazza PV, LeMoal M. Pathophysiological basis of vulnerability to drug abuse: Role of an interaction between stress, glucocorticoids, and dopaminergic neurons. Annual Review of Pharmacology and Toxicology 1996;36:359-378.

Porges SW. The polyvagal theory: Phylogenetic substrates of a social nervous system. International Journal of Psychophysiology 2001;42:123-146. [PubMed: 11587772]

Quevedo K, Benning SD, Gunnar MR, Dahl RE. The onset of puberty: Effects on the psychophysiology of defensive and appetitive motivation. Development and Psychopathology 2009;21:27-45. [PubMed: 19144221]

Radley JJ, Arias CM, Sawchenko PE. Regional differentiation of the medial prefrontal cortex in regulating adaptive responses to acute emotional stress. The Journal of Neuroscience 2006;26:12967-12976. [PubMed: 17167086] 
Roemmich JN, Richmond EJ, Rogol AD. Consequences of sport training during puberty. Journal of Endocrinological Investigation 2001;24:708-715. [PubMed: 11716157]

Rosen BR, Schulkin J. From normal fear to pathological anxiety. Psychological Review 1998;105:325-350. [PubMed: 9577241]

Silk JS, Siegel GJ, Whalen DJ, Ostapenko LJ, Ladouceur CD, Dahl RE. Pubertal changes in emotional information processing: Pupillary, behavioral, and subjective evidence during emotional word identification. Development and Psychopathology 2009;21:7-26. [PubMed: 19144220]

Sisk CL, Schulz KM, Zehr JL. Puberty: A finishing school for male social behavior. Annals of the New York Academy of Sciences 2003;1007:189-198. [PubMed: 14993053]

Sisk CL, Zehr JL. Pubertal hormones organize the adolescent brain and behavior. Frontiers in Neuroendocrinology 2005;26:163-174. [PubMed: 16309736]

Spear LP. The adolescent brain and age-related behavioral manifestations. Neuroscience and Behavioral Physiology 2000;24:417-463.

Spear, LP. Neurodevelopment during adolescence. In: Cicchetti, D.; Walker, EF., editors. Neurodevelopmental mechanisms in psychopathology. Cambridge University Press; Cambridge: 2003. p. 62-83.

Steinberg, L.; Dahl, R.; Keating, D.; Kupfer, DJ.; Masten, AS.; Pine, DS. The study of developmental psychopathology in adolescence: Integrating affective neuroscience with the study of context. In: Cicchetti, D.; Cohen, DJ., editors. Developmental neuroscience. Vol. 2. Wiley; New York: 2006. p. 710-741.

Stroud L, Foster E, Handwerger K, Papandonatos GD, Granger D, Kivlighan KT, et al. Stress response and the adolescent transition: Performance versus peer rejection stress. Development and Psychopathology 2009;21:47-68. [PubMed: 19144222]

Walker EF, Sabuwalla Z, Huot R. Pubertal neuromaturation, stress sensitivity, and psychopathology. Development and Psychopathology 2004;16:807-824. [PubMed: 15704816]

Waylen A, Wolke D. Sex 'n' drugs 'n' rock 'n' roll: The meaning and social consequences of pubertal timing. European Journal of Endocrinology 2004;151:151-159. [PubMed: 15248836]

West P. Health inequalities in the early years: Is there equalisation in youth? Social Science \& Medicine 1997;44:833-858. [PubMed: 9080566]

Zehr JL, Culbert KM, Sisk CL, Klump KL. An association of early puberty with disordered eating and anxiety in a population of undergraduate women and men. Hormones and Behavior 2007;52:427435. [PubMed: 17707381] 